Uso de los materiales didácticos en el aprendizaje significativo de los estudiantes Educación

DOI: $10.23857 /$ dc.v5i3.964

Ciencias de la educación

Artículo de investigación

\title{
Uso de los materiales didácticos en el aprendizaje significativo de los estudiantes Educación Básica
}

\section{Use of the didactic materials in the significant learning of the students Basic Education}

\section{Uso dos materiais didáticos na aprendizagem significativa dos alunos}

\author{
Carlos Enrique Santos-Loor ${ }^{\mathrm{I}}$ \\ carlossantosloor@hotmail.com \\ Carlina Patricia Santos-Loor ${ }^{\text {II }}$ \\ pasalo1966@hotmail.com
}

Hugo Jesús Juan Vélez-Pincay III

hvelez99@hotmail.com
Christian Alfredo Cevallos-Arteaga IV

cristhicevall@hotmail.com

María Vanesa Zamora-Lucas $\mathrm{v}$ vane_zamora_lucas@hotmail.es

Recibido: 23 de mayo de 2019 *Aceptado: 25 de junio de $2019 *$ Publicado: 05 de julio de 2019

I. Magíster en Docencia e Investigación Educativa, Doctor en Ciencias de la Educación Especialización Historia y Geografía, Licenciado en Ciencias de la Educación Especialización, Docente de la Facultad de Filosofía, Letras y Ciencias de la Universidad Técnica de Manabí, Portoviejo, Ecuador.

II. Magíster en Desarrollo Educativo, Doctora en Ciencias de la Educación Mención Investigación Educativa, Licenciada en Ciencias de la Educación Especialidad Psicología y Orientación Vocacional, Docente de la Facultad de Filosofía, Letras y Ciencias de la Universidad Técnica de Manabí, Portoviejo, Ecuador.

III. Magíster en Educación y Desarrollo Social, Licenciado en Ciencias de la Educación Especialización Química y Biología, Profesor de Segunda Enseñanza Especialización Química y Biología, Docente de la Facultad de Filosofía, Letras y Ciencias de la Universidad Técnica de Manabí, Portoviejo, Ecuador.

Iv. Maitrise Universitaire en Sciences de L'education - Analyse et Intervention dans les Systemes Educatifs, Baccellierato in Teologia, Docente de la Facultad de Filosofía, Letras y Ciencias de la Universidad Técnica de Manabí, Portoviejo, Ecuador.

v. Licenciada en Ciencias de la Educación Mención Educación Primaria, Docente Unidad Educativa Fiscal Franklin Delano Roosevelt, Portoviejo, Ecuador. 


\section{Resumen}

El propósito de este estudio es develar la percepción que tienen los docentes de la Unidad Educativa Costa Azul, en torno al uso de materiales didácticos para el aprendizaje significativo de los estudiantes de educación básica de esa institución, donde se ha observado durante las pasantías profesionales algunas debilidades dado el poco uso de este recurso. Los postulados teóricos se sustentan en Santibáñez (2006) y Ausubel (1978). La investigación se abordó desde el paradigma cualitativo a través del método hermenéutico interpretativo, desarrollando la técnica de entrevista en profundidad y como instrumento el guion de entrevista. Los hallazgos revelan una serie de códigos agrupados en cuatro (4) categorías: Instrumento educativo, Herramienta Instruccional, Construcción del Pensamiento y Relación objeto-sujeto. Las evidencias recogidas destacan limitaciones en cuanto al compromiso institucional y profesional, lo cual dificulta el ejercicio de esta praxis pedagógica, pero también la asunción de lo importante que es la interacción de manera más activa y lúdica del uso de materiales didácticos para fomentar los aprendizajes de manera que el estudiante incremente su desarrollo cognitivo, sensorial y psicomotor.

Palabras Claves: Materiales didácticos; aprendizaje significativo.

\section{Abstract}

The purpose of this study is to reveal the perception that teachers have of the Costa Azul Educational Unit, about the use of didactic materials for meaningful learning of basic education students of that institution, where some professional internships have been observed Weaknesses given the little use of this resource. The theoretical postulates are supported by Santibáñez (2006) and Ausubel (1978). The research was approached from the qualitative paradigm through the interpretive hermeneutic method, developing the technique of in-depth interview and as instrument the interview script. The findings reveal a series of codes grouped into four (4) categories: Educational Instrument, Instructional Tool, Construction of Thought and Object-Subject Relationship. The collected evidence highlights limitations in terms of institutional and professional commitment, which makes it difficult to exercise this pedagogical practice, but also the assumption of how important interaction is in a more active and playful way of using teaching materials to promote learning from so that the student increases their cognitive, sensory and psychomotor development.

Keywords: Didactic materials; significant learning.

775 Vol. 5, núm. 3,julio, 2019, pp.774-783 Carlos Enrique Santos Loor, Carlina Patricia Santos Loor, Hugo Jesús Juan Vélez Pincay, Christian Alfredo Cevallos Arteaga, María Vanesa Zamora Lucas 


\section{Resumo}

O objetivo deste estudo é revelar a percepção dos professores na Unidade de Educação Costa Azul, sobre o uso de materiais de ensino para a aprendizagem significativa de alunos do ensino fundamental dessa instituição, que tem sido observado durante algum estágios profissionais Fraquezas dado o pouco uso deste recurso. Os postulados teóricos são baseados em Santibanez (2006) e Ausubel (1978). A pesquisa foi abordado a partir do paradigma qualitativo através do método hermenêutico interpretativo, desenvolvendo técnica de entrevista em profundidade e como instrumento do roteiro de entrevista. Os resultados revelam uma série de códigos agrupados em quatro (4) categorias: Educacional Instrumento Instrucional ferramentas, Construção pensamento e de relacionamento sujeito-objeto. Os dados recolhidos incluem limitações sobre o compromisso institucional e profissional, o que dificulta o exercício dessa práxis pedagógica, mas também a assunção da importância da interação de forma mais activa e divertida de usar materiais de ensino para promover a aprendizagem de para que os alunos aumentar o seu desenvolvimento cognitivo, sensorial e psicomotora.

Palavras-chave: materiais didáticos; aprendizagem significativa.

\section{Introducción}

Cuando se trata de procesos de formación en la primera infancia, el docente debe proveerse de recursos educativos inherentes al impulso biopsicosocial y cognitivo del niño. Al hacer alusión de estos elementos, se habla en específico de materiales didácticos que proporcionen el desarrollo psicomotor, perceptivo, socio-afectivo y del lenguaje, áreas que se organizan en concordancia a la edad biológica, poseedoras de características particulares dada la complejidad individual de cada sujeto.

De allí que, el Fondo de las Naciones Unidas para la Infancia (UNICEF: 2018), se planteara dentro de una de sus metas de desarrollo sostenible, asegurar que de aquí al 2030 se haya conseguido el máximo de aprendizaje temprano para los niños a fin de descubrir su potencial como estrategia clave para mejorar el aprendizaje y la calidad educativa.

En este contexto, Piaget (1959) expresa la importancia del aprendizaje temprano para la evolución intelectual que comienza en la primera etapa de la vida, por ello se debe seleccionar muy bien los

776 Vol. 5, núm. 3,julio, 2019, pp.774-783 Carlos Enrique Santos Loor, Carlina Patricia Santos Loor, Hugo Jesús Juan Vélez Pincay, Christian Alfredo Cevallos Arteaga, María Vanesa Zamora Lucas 
recursos que contribuirán al cumplimiento de esta tarea ya que es en esta edad donde los infantes absorben el mayor número de información y requieren de estímulos externos para procesar las experiencias que están adquiriendo.

La pedagogía actual cuenta con una diversidad de elementos didácticos para poner al servicio de los estudiantes; por eso es menester de los docentes utilizarlos en la construcción de aprendizajes significativos en la transmisión de los nuevos saberes y así proveerle la posibilidad de tener un mejor desarrollo en todas sus dimensiones, a saber: biológicas, psicológicas, físicas y mentales.

Sin embargo, es evidente la carencia de estos elementos dentro del aula y en el desempeño de la labor educativa, lo que incide en el rendimiento académico y desencadena el desinterés, la desmotivación y la apatía; practicas pedagógicas arraigadas a los modelos tradicionales, limitados al salón de clases, el marcador de pizarra, y la información unidireccional. Por tal razón, surge la siguiente interrogante: ¿Cuál es la percepción que poseen los docentes de la Unidad Educativa Costa Azul, en torno al uso de materiales didácticos para el aprendizaje significativo de los estudiantes del segundo año de educación básica?

En tal sentido, se plantea como propósito de la investigación develar la percepción que tienen los docentes de la Unidad Educativa Costa Azul, en torno al uso de materiales didácticos para el aprendizaje significativo de los estudiantes del segundo año de educación básica, mediante la hermenéutica como método de indagación.

\section{Desarrollo de las categorías en estudio}

El material didáctico, según Santibáñez (2006), es todo aquel instrumento que posibilita al docente realizar experiencias educativas, formativas e informativas manejando los objetos, seres y fenómenos para relacionarlos con el contexto en el que desarrolla su praxis pedagógica, por tanto, deben formarse apropiadamente para conducir y asesorar a sus estudiantes durante el aprendizaje.

En palabras de Cebrián (1992:158), para poner en práctica esta estrategia educativa se pueden utilizar objetos, equipos y aparatos tecnológicos, así como espacios y lugares de interés cultural, programas o itinerarios medio ambientales, materiales educativos que son referentes directos de la realidad y

\footnotetext{
777 Vol. 5, núm. 3,julio, 2019, pp.774-783 Carlos Enrique Santos Loor, Carlina Patricia Santos Loor, Hugo Jesús Juan Vélez Pincay, Christian Alfredo Cevallos Arteaga, María Vanesa Zamora Lucas
} 
enlazan las diversas formas de representación simbólica, la reconstrucción del conocimiento y los significados culturales del currículum.

Por su parte, Moreno (2013:330), considera el material educativo como un elemento que ha sido creado con un fin pedagógico; es decir, una herramienta académica útil y necesaria para incentivar, ampliar y concretar la construcción del conocimiento, mediante el proceso de enseñanza y aprendizaje utilizado como estrategia didáctica en apoyo a los docentes y en provecho de los estudiantes.

Con relación al aprendizaje significativo, Ausubel (1976:56) refiere que es un proceso con intenciones y orientaciones específicas para posibilitar la constitución de vínculos sustantivos y no arbitrarios de los nuevos contenidos que se ha de aprender y aquellos que se encuentran en la estructura cognitiva del estudiante, cuya esencia reside en que las ideas expresadas simbólicamente al ser relacionadas de manera sustancial y no al pie de la letra.

\section{El Método}

En cuanto al método hermenéutico abordado en el presente trabajo, García (2000), lo define como, el arte de descifrar el sentido de las acciones del hombre, de comprender sus textos literarios, sus comportamientos éticos y sus creaciones estéticas. La aplicación de este método, le permitirá al investigador aproximarse a la realidad de la institución, el docente y los estudiantes, en torno al uso del material didáctico y así poder recoger información, a través del cumplimiento de todo el proceso metodológico mediante el ejercicio exegético sobre la interpretación de las opiniones de lo que piensan los actores involucrados como sujetos investigados.

En concordancia con el método expuesto, se aplicó como técnica cualitativa la entrevista en profundidad, definida por Veliz (2009) como un proceso de interacción dinámica de comunicación entre 2 personas, es decir el entrevistador y el entrevistado, controlado por el primero, donde este busca obtener información lo más completa y detallada posible en el tema planteado. En lo que respecta al instrumento, fue un guion de entrevista, aplicado a dos docentes de la escuela como actores sociales de la investigación, cuyo escenario fue la Unidad Educativa Costa Azul, ubicado en la ciudad de Manta, Ecuador.

\footnotetext{
778 Vol. 5, núm. 3,julio, 2019, pp.774-783

Carlos Enrique Santos Loor, Carlina Patricia Santos Loor, Hugo Jesús Juan Vélez Pincay, Christian Alfredo Cevallos Arteaga, María Vanesa Zamora Lucas
} 


\section{Hallazgos y categorías subyacentes de la hermenéutica aplicada}

Las valoraciones acerca de la realidad, del uso de materiales didácticos para incentivar el aprendizaje significativo de los estudiantes del segundo año de básica en la Unidad Educativa Costa Azul, son presentadas mediante conceptualizaciones de las categorías emergentes del material de las entrevistas.

Dichas conceptualizaciones, se generan con base a la interpretación de las experiencias de los informantes que fueron ubicadas en cada categoría. Luego de conocer los conceptos emergentes, se sintetiza textual y gráficamente las opiniones de los entrevistados, lo cual permite al investigador establecer las reflexiones pertinentes.

En primer lugar subyace la categoría Instrumento educativo: Se constituye como un elemento esencial en el que se apoya el docente para proyectar las bases en las que se cimienta lo que desea enseñar. Las opiniones que dan origen a la identificación de esta categoría son:

- "Es que aquí no tenemos como trabajar, la institución provee de muy pocos materiales, para poder utilizarlos hacerlo debemos construirlos nosotros mismos"

- "Lo que pasa es que el contenido programático del currículo es tan absorbente que no me permite sino ir directo con los recursos tradicionales para no perder el tiempo"

- "Se puede utilizar como apoyo si pero es muy agotador".

Aunque los docentes reconocen la utilidad de los materiales didácticos, algunos de ellos consideran que realizar esa práctica les acarrea más trabajo, por ende manejan solo los recursos puntuales sin demostrar iniciativa ni creatividad en el acto pedagógico, hecho que merma su rol como mediador del aprendizaje y formador en detrimento de la calidad educativa.

Seguidamente, surge Herramienta Instruccional: Está compuesta por una serie de mecanismos que regulan el proceso de instrucción en la contribución de la dinámica que sugiere el acto pedagógico. Las expresiones que soportan esta categoría son:

- “Cuando les muestro el material didáctico se animan y es más fácil para ellos entender"

779 Vol. 5, núm. 3,julio, 2019, pp.774-783 Carlos Enrique Santos Loor, Carlina Patricia Santos Loor, Hugo Jesús Juan Vélez Pincay, Christian Alfredo Cevallos Arteaga, María Vanesa Zamora Lucas 
- "Me propongo utilizar materiales didácticos al menos una vez a la semana porque a ellos les encanta y prestan más atención por la novedad"

- "Si, es un medio que me permite conectarlos con el contenido educativo y por eso la incluyo en mi planificación"

Si bien es cierto que para algunos docentes el uso de material didáctico no es necesario; no es menos cierto que para la mayoría es de suma importancia vista la utilidad y envergadura de estos, los cuales repercuten en el desarrollo evolutivo del niño e inciden directamente en la apropiación del conocimiento para aprender, analizar y evaluar las cosas.

La otra categoría emergida fue Construcción del Pensamiento: Es la fabricación de conceptos concernientes al aprendizaje relacionados con las experiencias obtenidas durante el proceso de aprehensión y abstracción. Al respecto, los informantes manifestaron:

- $\quad$ "Ellos se forman una idea de las cosas y hacen muchas preguntas cuando manipulan el material didáctico"

- "Su actitud y cambia definitivamente cada vez que les informo que los vamos a utilizar, por eso los considero muy provechoso, además son muy divertidos, porque aprenden jugando en la interacción con los objetos y les motiva a la participación”

- "Mis niños se ponen muy feliz cuando les saco cualquier material didáctico, eso les despierta el interés"

Para los niños, la creación de conceptos es su propia obra donde expresan el significado que para ellos tienen las cosas, dando rienda suelta a la creatividad y la imaginación porque se les permite jugar mientras aprenden y obtienen con claridad la visión y percepción de los objetos haciendo explicitas sus conjeturas y juicios valorativos de lo que denota y connota el objeto de estudio.

Por último, germina la categoría Relación objeto-sujeto: Se considera como la iniciación epistemológica en la que el sujeto se encuentra inmerso, una vez que ha podido vincular la esencia de

780 Vol. 5, núm. 3,julio, 2019, pp.774-783 Carlos Enrique Santos Loor, Carlina Patricia Santos Loor, Hugo Jesús Juan Vélez Pincay, Christian Alfredo Cevallos Arteaga, María Vanesa Zamora Lucas 
las cosas aprendidas con los juicios que ya trae o los que se ha formado en el momento, tal y como lo refieren los entrevistados:

- "Los niños cuando están con los materiales didácticos aprenden sin presión y esa flexibilidadles ayuda a clasificar, comparar y analizar las cosas mejor"

- "Los estudiantes hacen una representación simbólica y se adhieren a la conexión figurada de su imaginario"

- "El conocimiento lo obtienen de forma natural de acuerdo a su realidad empírica y razonamiento".

El carácter racional y cognoscente con la que el niño aprehende las cosas les permite hacer inferencias a través de la estructura del pensamiento desde una racionalidad lógica porque parte de supuestos creados en su mente mediante un acto de intelección para producir conocimiento, factor preponderante para reafirmar el entorno con el que se está relacionando.

Valoración final de la percepción que tienen los docentes sobre el uso de materiales didácticos para el aprendizaje significativo.

Las reflexiones en torno a las circunstancias generales de cómo se lleva a el uso de material didáctico y su incidencia en el aprendizaje significativo de los estudiantes del segundo año de básica por parte del investigador y la información recolectada a través de entrevistas realizadas al personal docente de la institución, permitieron develar las particularidades institucionales y profesionales relacionados con esta praxis pedagógica.

Con base a las consideraciones que anteceden, se observa que algunos de los recursos trabajados dentro del aula de clase y catalogados como materiales didácticos son un gran medio lúdico y dinamizador para el proceso de enseñanza y aprendizaje, lo cual permite despertar el interés de los estudiantes en la medida en que se alejan del aburrimiento y la rutina, por cuanto configura una forma de aprender jugando. Además, contribuye al manejo de herramientas que coadyuvan a la construcción del conocimiento.

\footnotetext{
781 Vol. 5, núm. 3,julio, 2019, pp.774-783 Carlos Enrique Santos Loor, Carlina Patricia Santos Loor, Hugo Jesús Juan Vélez Pincay, Christian Alfredo Cevallos Arteaga, María Vanesa Zamora Lucas
} 
En virtud de ello, urge la necesidad de que el docente dentro de su autonomía y compromiso vacacional como facilitador y mediador del aprendizaje, pueda orientar y formar ciudadanos mientras innova en el acto pedagógico y transfiere sus enseñanzas a medida que sus participantes demuestran la apropiación de las nociones elementales académicas de una manera más práctica y cercana a la realidad del contexto.

Con este ejercicio, no solo se revelan los conocimientos del maestro y su capacidad sino también las competencias desarrolladas en la gestión educativa para adaptar los contenidos curriculares a los procesos y ritmos de los niños, al promover en ellos el desarrollo sensorial y psicomotor, mientras incrementa sus deseos por conocer, hacerse preguntas, explorar el medio que les rodea e ir más allá de lo que proporciona el docente cuando hace la conexión de los objetos, hechos o cosas utilizadas en el salón o en espacios abiertos para edificar sus propios saberes y adquirir las habilidades requeridas en su proceso formativo. 


\section{Referencias Bibliográficas}

Ausubel, David P. (1976) Descripción: Psicología educativa. Un punto de vista cognitivo. México: Editorial Trillar, p. 769.

Cebrián De La Serna, M. (1992). La didáctica, el currículo, los medios y los recursos didácticos. Málaga: Secretariado de Publicaciones, Universidad de Málaga.

García J. (2000) Ética y hermenéutica. Editorial Nueva Madrid.

Moreno, F. (2013). La manipulación de los materiales como recurso didáctico en educación infantil Universidad Católica San Antonio de Murcia Estudios sobre el Mensaje Periodístico 331. Vol. 19 Núm. especial 329337.

Piaget, J. (1959). La formación del símbolo en el niño. México: Fondo de Cultura Económica.

Santibáñez, V. (2006). Un enfoque renovado del material didáctico. 1ra edición. Perú: Editorial IMACHI SRL.

UNICEF, Fondo de las Naciones Unidas para la Infancia (2018). Aprendizaje a través del juego. Sección de Educación, División de Programas 3 United Nations Plaza, New York, NY 10017.

Veliz C. (2009) Proyectos Comunitarios e Investigación Cualitativa. Caracas Venezuela: Editorial Texto, C.A

Vygotsky, Lev Semiónovich. (1979): El desarrollo de los procesos psicológicos superiores. Barcelona, Crítica. 